\title{
Development of Laboratory-type Soft X-ray Microscope, CXRM, in Water-Window using LPP Light Source
}

Ejima, Takeo ${ }^{1,}{ }^{*}$, Ono, Yuichi ${ }^{2}$, Ito, Keisuke ${ }^{2}$, Kawasaki, Hiromu $^{2}$, Higashiguchi, Takeshi $^{2}$, Tone, Shigenobu ${ }^{3}$, and Hatano, Tadashi ${ }^{1}$

1. IMRAM, Tohoku University, Sendai, JAPAN

2. Dept. of Elec. \& Elec. Engineer., Utsunomiya University, Utsunomiya, JAPAN

3. Sch. of Sci \& Technol., Tokyo Denki University, Hatoyama, JAPAN

* Corresponding author, takeo.ejima.e7@tohoku.ac.jp

In biological and its related sciences, the demands of high-spatial-resolution observation are increasing, and the demands are suggested by that the recent development of some super resolution techniques with luminescent staining methods. The luminescent staining methods are also used to identify organelles in a bio-cell and are standard techniques for the identification of the organelles [1]. X-ray microscopy is recently developed because it shows the high spatial resolution without any staining of organelles. On the other hand, the organelles observed in X-ray microscope images cannot be exactly identified because there is no identification information. One of the solutions to identify the organelles is to combine of the usual staining-methods for organelle-identification with X-ray microscopy. In this study, an instrument capable of both visible and X-ray was constructed.

The developed microscope is a contact-type X-ray microscope (CXRM), the optical layout of the microscope is represented in Figure 1. CXRM is composed of a laser produced plasma (LPP) light source, a soft X-ray (SX) condenser optics including a scintillator plate that converts a SX image into a visible image, and an optical microscope which magnifies the visible image. The light source is composed of a Bi target, and a Nd:YAG laser $(\lambda=1064 \mathrm{~nm}$, duration time $=150 \mathrm{ps},<250 \mathrm{~mJ} / \mathrm{pulse}, 10$ $\mathrm{Hz})$ as an excitation laser. The SX light is produced in the water window wavelength range $(2.4 \mathrm{~nm}-$ $4.4 \mathrm{~nm}$ ). The emission spectrum is represented as a broken curve in Figure 2 [2]. The SX light is collected by the condenser optics which is composed of two toroidal mirrors. The design of the toroidal mirror is reported elsewhere [3]. The mirror surface is coated with a $\mathrm{Sc} / \mathrm{Cr} / \mathrm{Mo}$ reflection multilayer designed to reflect the Bi emission $[2,4]$. The reflectance spectra of the different positions on the mirror surface are presented by solid curves in Figure 2. The peak positions of the reflectance peaks were calibrated by the periodic length of the reflection multilayer to fit the peak position of the $\mathrm{Bi}$ emission peak $(\lambda=4.1 \mathrm{~nm})$ during the multilayer deposition. Small reflection peaks around $4.4 \mathrm{~nm}$ are
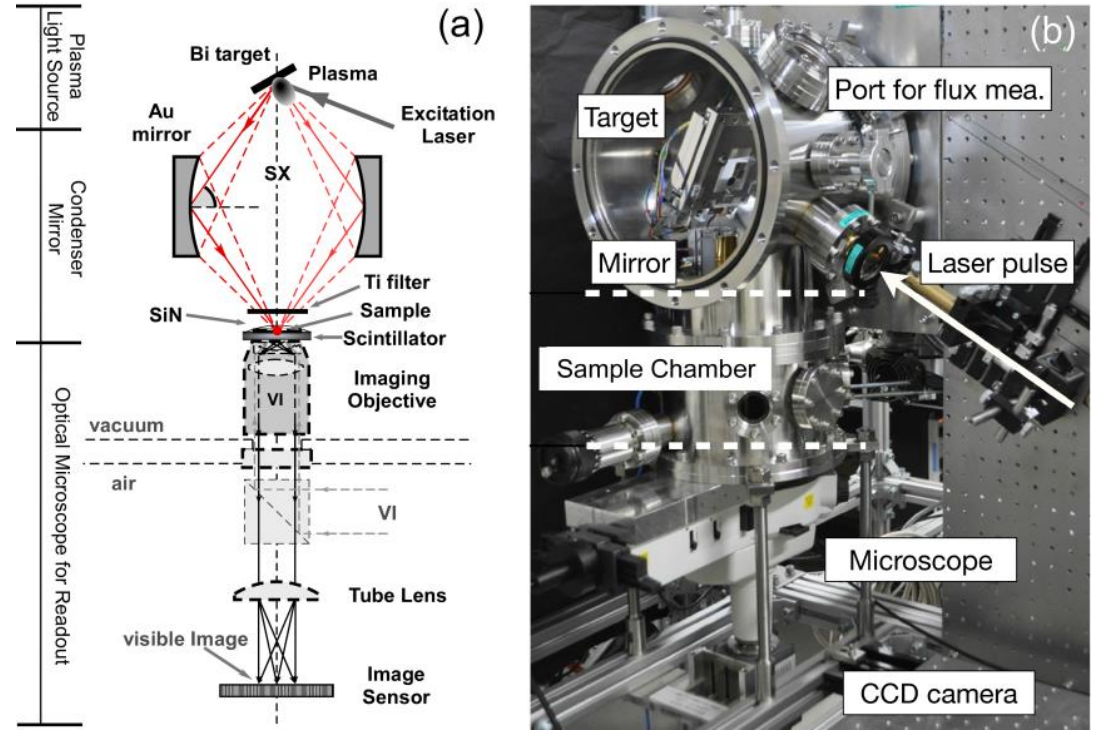

Figure 1. Optical layout of the CXRM (a), and the details of the developed microscope (b). 
the error of the measurement which originates from the contamination of the beamline optics used in the reflectance measurements. An optical filter is placed along the optical path to cut the visible light from the LPP light source. The filter is a $100 \mathrm{~nm}$ thick Ti membrane. Combining the reflectance of the reflection multilayer with the transmittance of the filter, the light at the water window wavelength range passes through the optics and focuses on a sample, which is placed on a scintillator plate. Transmitted SX light through the sample is converted to visible light by the scintillator plate. The scintillator used in the conversion is Ce:LSO which shows high quantum efficiency and linearity in the water window wavelength range [5]. Thus, SX images can be observed instantly by the visible microscope (LV150N, Nikon Instruments Inc., objective magnification is $50 \times$ ), and can be directly compared with visible-light images as the result of illumination-source change.

To demonstrate the performance of the developed microscope, Hela S3 cells cultured on a scintillator plate were observed. Images of several cells are represented in Figure 3. The image is a summation of 500 shots of the laser pulse (exposure time is $50 \mathrm{sec}$ ). The cell that is indicated by an arrow shows that a nucleus is in the center of the cell, small white structures surround the nucleus, and a structure like a thin membrane is observed outside of the cell. Bright edge around the nucleus may be an artifact caused by the sample preparation. The present status of CXRM is that in-situ observation of both visible and X-ray lights is possible.

\section{References:}

[1] B. Alberts, et al., "Molecular Biology of The Cell $6^{\text {th }}$ Ed.", (Garland Science, 2014, New York) Chap. 9.

[2] T. Higashiguchi, et al., Appl. Phys. Lett. 100, (2012) 014103.

[3] T. Ejima, et al., Appl. Optics 53, (2014) 68466852.

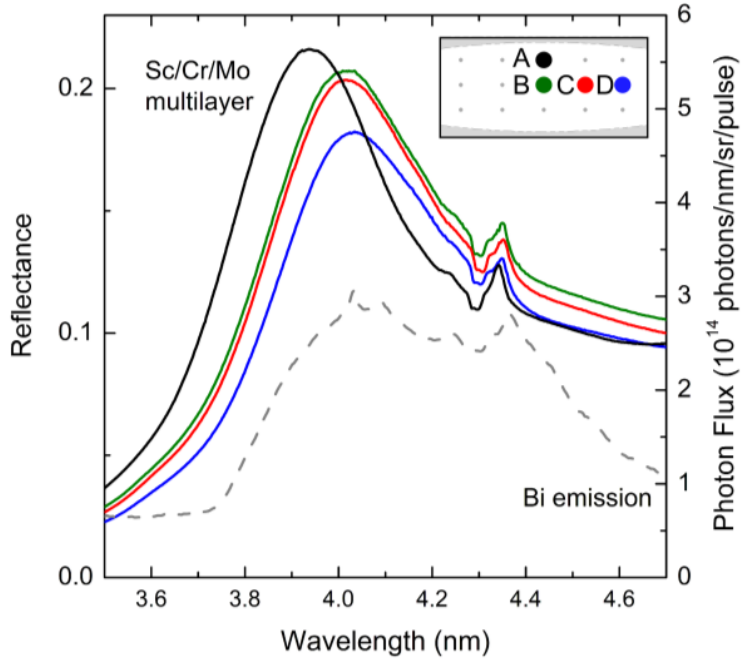

Figure 2. Reflectance of the $\mathrm{Sc} / \mathrm{Cr} / \mathrm{Mo}$ multilayer coated on the toroidal mirror at some positions of the mirror as designated in the inset (solid curves), and brilliance from the Bi emission (broken curve).

[4] T. Hatano, et al., J. Elec. Spec. Rel. Phenom. 220, (2017) 14-16.

[5] T. Ejima, et al., J. Phys.: Conf. Series 463, (2013) 012055.

[6] This work was supported by Grants-in-Aid for Scientific Research (JSPS Kakenhi, Grant Number: 23241038, 16H03902, and 17K190210). Mr. Y. Nakamura belonging to the workshop of IMRAM, Tohoku Univ. is thanked for polishing scintillators.

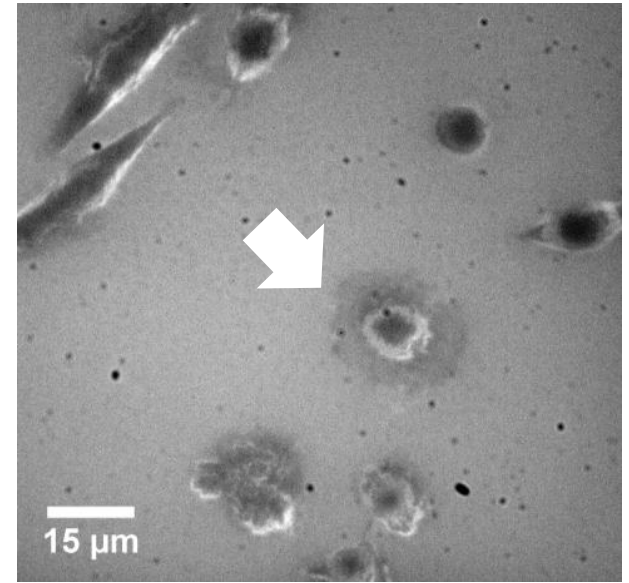

Figure 3. HeLa S3 cells cultured on a scintillator plate. Small dots are debris in the optical path. 